\title{
On the energy-momentum tensor in Moyal space
}

\author{
Herbert Balasin ${ }^{1, \mathrm{a}}$, Daniel N. Blaschke ${ }^{2, \mathrm{~b}}$, François Gieres ${ }^{3, \mathrm{c}}$, Manfred Schweda ${ }^{1, \mathrm{~d}}$ \\ ${ }^{1}$ Institute for Theoretical Physics, Vienna University of Technology, Wiedner Hauptstraße 8-10, 1040 Vienna, Austria \\ 2 Theory Division, Los Alamos National Laboratory, Los Alamos, NM 87545, USA \\ ${ }^{3}$ Institut de Physique Nucléaire de Lyon, Bat. P. Dirac, Université de Lyon, Université Claude Bernard Lyon 1 and CNRS/IN2P3, \\ 4 rue Enrico Fermi, 69622 Villeurbanne, France
}

Received: 18 February 2015 / Accepted: 29 May 2015 / Published online: 26 June 2015

(C) The Author(s) 2015. This article is published with open access at Springerlink.com

\begin{abstract}
We study the properties of the energy-momentum tensor of gauge fields coupled to matter in non-commutative (Moyal) space. In general, the non-commutativity affects the usual conservation law of the tensor as well as its transformation properties (gauge covariance instead of gauge invariance). It is well known that the conservation of the energymomentum tensor can be achieved by a redefinition involving another star-product. Furthermore, for a pure gauge theory it is always possible to define a gauge invariant energymomentum tensor by means of a Wilson line. We show that the last two procedures are incompatible with each other if couplings of gauge fields to matter fields (scalars or fermions) are considered: The gauge invariant tensor (constructed via Wilson line) does not allow for a redefinition assuring its conservation, and vice versa the introduction of another starproduct does not allow for gauge invariance by means of a Wilson line.
\end{abstract}

\section{Contents}

1 Introduction . . . . . . . . . . . . 1

2 EMT for a gauge field in Moyal space . . . . . . . 2

3 Coupling to neutral matter fields . . . . . . . . . . . 3

3.1 Complex scalar field . . . . . . . . . . . 3

3.2 Fermions . . . . . . . . . . . 6

4 Coupling to charged matter fields $\ldots \ldots$. . . . . 7

4.1 Complex scalar field . . . . . . . . . . 7

4.1.1 Fundamental representation . . . . . . 7

4.1.2 Antifundamental representation . . . . 8

4.2 Fermions . . . . . . . . . . . 8

4.2.1 Fundamental representation . . . . . 8

\footnotetext{
a e-mail: hbalasin@tph.tuwien.ac.at

be-mail: dblaschke@lanl.gov

c e-mail: gieres@ipnl.in2p3.fr

de-mail: mschweda@tph.tuwien.ac.at
}

4.2.2 Antifundamental representation . . . . . 9

5 Conclusion . . . . . . . . . . . . . . 9

References . . . . . . . . . . . . . . . 10

\section{Introduction}

Groenewold-Moyal (or $\theta$-deformed) space [1,2] represents one of the simplest models for quantized spaces, and has been extensively studied over the past 20 years; e.g. see [3-5] and references therein for a review. The theories on this space are formulated in terms of ordinary functions by means of a deformed product, the so-called star-product,

$(f \star g)(x)=\left.\mathrm{e}^{\frac{\mathrm{i}}{2} \theta^{\mu \nu} \partial_{\mu}^{x} \partial_{\nu}^{y}} f(x) g(y)\right|_{y=x}$,

which implies that the coordinates fulfill

$\left[x_{\mu} \stackrel{\star}{,} x_{\nu}\right] \equiv x_{\mu} \star x_{v}-x_{v} \star x_{\mu}=\mathrm{i} \theta_{\mu \nu}$.

This commutation relation is invariant under translations of the space-time coordinates and under the so-called reduced Lorentz transformations (or reduced orthogonal transformations in the Euclidean setting); see for instance Ref. [6].

In general, field theoretic models on such spaces suffer from a new type of divergences which arise due to a phenomenon referred to as UV/IR mixing $[7,8]$ and which render the models non-renormalizable. This problem can be overcome in the case of some special scalar field models [9-11], one of them having been shown to be solvable even nonperturbatively [12].

The present work is devoted to a basic aspect of classical field theories on Moyal space, namely the energy-momentum tensor (hereafter referred to as EMT) and its properties at tree level. In earlier studies [13-18] some modifications to the conservation law of the EMT due to the non-commutativity parameters $\theta^{\mu \nu}$ were found in $\phi^{\star 4}$ and in gauge theory (without matter couplings). Here, we wish to investigate more generally complex scalars and fermions coupled to $U_{\star}(1)$ gauge 
fields. In view of the infamous time-ordering problems in quantum field theory on Moyal space [19], we restrict ourselves to the Euclidean version of Moyal space.

The present work is organized as follows. In Sect. 2, we examine the gauge invariance and conservation properties of the EMT for a gauge field in Moyal space. In Sects. 3 and 4 we then extend the discussion to include various couplings to matter.

\section{EMT for a gauge field in Moyal space}

We consider a $U_{\star}(1)$ gauge field $\left(A^{\mu}\right)$ coupled to an external current $\left(J^{\mu}\right)$ in four-dimensional flat Euclidean ${ }^{1}$ Moyal space: the action

$S[A]=\frac{1}{4} \int \mathrm{d}^{4} x F_{\mu \nu} \star F^{\mu \nu}+\int \mathrm{d}^{4} x J^{\mu} \star A_{\mu}$,

with $F_{\mu \nu}=\partial_{\mu} A_{\nu}-\partial_{\nu} A_{\mu}-\mathrm{i} g\left[A_{\mu} \stackrel{\star}{,} A_{\nu}\right]$,

yields the equation of motion

$0=\frac{\delta S[A]}{\delta A_{v}}=-D_{\mu} F^{\mu v}+J^{v}$

The functional $S[A]$ is invariant under the infinitesimal gauge transformations

$$
\begin{gathered}
\delta_{\lambda} A_{\mu}=D_{\mu} \lambda \equiv \partial_{\mu} \lambda-\mathrm{i} g\left[A_{\mu}{ }^{\star}, \lambda\right], \\
\delta_{\lambda} F_{\mu \nu}=-\mathrm{i} g\left[F_{\mu \nu}, \lambda\right],
\end{gathered}
$$

provided the current $\left(J^{\mu}\right)$ does not transform and is covariantly conserved, i.e. $D_{\mu} J^{\mu}=0$. This is also consistent with the equation of motion in the sense that

$$
\begin{aligned}
D_{\nu} J^{\nu}=D_{\nu}\left(D_{\mu} F^{\mu \nu}\right) & =\frac{1}{2}\left[D_{\nu}, D_{\mu}\right] F^{\mu \nu} \\
& =\frac{\mathrm{i} g}{2}\left[F_{\mu \nu}, F^{\mu \nu}\right]=0 .
\end{aligned}
$$

Notice, however, that gauge invariance of the equation of motion (4) requires that $J^{\mu}$ transforms covariantly, i.e. $\delta_{\lambda} J^{v}=-\mathrm{i} g\left[J^{v}{ }^{\star}, \lambda\right]$, but that would destroy gauge invariance of the action unless $\partial_{\mu} J^{\mu}=0$. This inconsistency was already noticed in Ref. [20] and is due to the non-Abelian nature of non-commutative gauge theory. In fact, a similar inconsistency occurs in Yang-Mills theory on ordinary commutative space when coupling the gauge field to an external current [21]. This problem can be overcome by coupling the

\footnotetext{
${ }^{1}$ We recall that certain signs (e.g. some global signs in the actions) change upon passage from Minkowskian to Euclidean signature. The coupling constant is denoted by $g$ and there should be no risk of confusion with the determinant of the metric tensor $\left(g_{\mu \nu}\right)$ considered for defining the Einstein-Hilbert EMT.
}

gauge field to dynamical complex scalar and/or fermion fields so that the external current is replaced by the corresponding matter current; see the next sections. For now, we keep in mind that the action (3) is not the complete action.

Concerning the transformation laws (5) we emphasize that, by contrast to a $U(1)$ gauge theory in ordinary Minkowski space, the field strength $F_{\mu \nu}$ is not a gauge invariant quantity as in electrodynamics. This non-Abelian nature of the theory in Moyal space is due to the noncommutativity of space-time coordinates, which implies that the field strength "feels" the non-commutativity of the space in which it lives. (This even applies to the simplest case of a constant field strength [22].) The transformation law of $F_{\mu \nu}$ implies that the Lagrangian density $\mathcal{L}=\frac{1}{4} F_{\mu \nu} \star F^{\mu \nu}$ is not invariant under gauge transformations since $\delta_{\lambda} \mathcal{L}=-\mathrm{i} g[\mathcal{L}$, $\lambda]$ : it is only the integral which plays the role of a trace which ensures cyclic invariance of factors and thereby gauge invariance. Henceforth, the lack of gauge invariance of the EMT will not come as a surprise and contrasts the situation for non-Abelian Yang-Mills fields in Minkowski space.

The improved EMT for a free (i.e. not coupling to a current) gauge field in Moyal space was already computed in Refs. [16-18]:

$T^{\mu \nu}=\frac{1}{2}\left(\left\{F^{\mu \rho \star}, F_{\rho}^{\nu}\right\}-\frac{1}{2} \delta^{\mu \nu} F_{\rho \sigma} \star F^{\rho \sigma}\right)$.

It is symmetric and traceless, and it transforms covariantly under gauge transformations:

$\delta_{\lambda} T^{\mu \nu}=-\mathrm{i} g\left[T^{\mu \nu} \star \lambda\right]$.

From the Bianchi identity $D_{\mu} F_{\nu \rho}+D_{\nu} F_{\rho \mu}+D_{\rho} F_{\mu \nu}=0$ and the equation of motion (4) with $J^{\mu}=0$, it follows that the covariant divergence of the gauge field EMT vanishes,

$D_{\mu} T^{\mu \nu}=0$,

i.e. $T^{\mu \nu}$ is covariantly conserved.

In the Minkowskian version of Moyal space with noncommutativity parameters satisfying $\theta^{0 i}=0$, the integral $\int \mathrm{d}^{3} x$ of a star-commutator vanishes (assuming as usual that fields vanish sufficiently fast at spatial infinity), hence Eq. (9) implies that

$$
\begin{aligned}
0= & \int \mathrm{d}^{3} x D_{\mu} T^{\mu v}=\int \mathrm{d}^{3} x \partial_{0} T^{0 v}=\frac{\mathrm{d} P^{v}}{\mathrm{~d} t}, \\
& \text { with } P^{v} \equiv \int \mathrm{d}^{3} x T^{0 v} .
\end{aligned}
$$

Thus, the four-momentum $\left(P^{v}\right)$ of the gauge field represents a conserved quantity. Moreover, this quantity is gauge invariant by virtue of (8) and the definition of $P^{v}$ in terms of $T^{0 v}$. 
Let us now come back to the local transformation law (8). In Ref. [23] (see also [24]) it was explained how to construct gauge invariant objects in Moyal space out of gauge covariant ones. In fact, this task is achieved by folding the quantity in question with a straight Wilson line defined by a length vector $\left(l^{\mu}\right)$ with $l^{\mu}=\theta^{\mu \nu} k_{v} \equiv(\theta k)^{\mu}$. Using this procedure, the authors of Ref. [16] obtained a standard local conservation law for the so constructed EMT. In the following we will also follow this strategy for gauge fields, scalars and fermions, and therefore we briefly outline the procedure here.

The non-commutative generalization of a straight Wilson line with the appropriate length is given by

$W(k, x)=\mathcal{P}_{\star} \exp \left(\int_{0}^{1} \mathrm{~d} \sigma A_{\mu}(x+\sigma \theta k) \theta^{\mu v} k_{\nu}\right)$,

where $\mathcal{P}_{\star}$ denotes path ordering with respect to the contour parameter $\sigma$. The expression (11) transforms as $W(k, x) \rightarrow$ $U(x) \star W(k, x) \star U(x+\theta k)^{\dagger}$ under a gauge transformation $U(x)$. Hence, $\int \mathrm{d}^{4} x W(k, x) \star \exp (\mathrm{i} k x)$ is a gauge invariant object because the length vector of the Wilson line is adjusted to be $\theta^{\mu \nu} k_{v}$ and $\exp (\mathrm{i} k x)$ induces a translation of $U^{\dagger}$ by $-\theta k$, cf. [23,25]. One may now construct (Fourier transforms of) gauge invariant objects from gauge covariant ones by star-multiplication with $W(k, x)$ and $\exp (\mathrm{i} k x)$ and integrating over $\mathrm{d}^{4} x$. The choice of a straight Wilson line is the most natural one because for such a line it makes no difference if the operator is attached to an endpoint of the Wilson line or somewhere in the middle [23]. Furthermore, in the commutative limit $(\theta \rightarrow 0)$ the Wilson line's length goes to zero.

For the EMT of a gauge field in Moyal space this means that

$\tilde{T}^{\mu \nu}(y) \equiv \int \frac{\mathrm{d}^{4} k \mathrm{~d}^{4} x}{(2 \pi)^{4}} \mathrm{e}^{\mathrm{i} k(y-x)} \star W(k, x) \star T^{\mu \nu}(x)$

is a gauge invariant quantity ${ }^{2}$ (which reduces in the commutative limit to the ordinary EMT due to $\left.\lim _{\theta \rightarrow 0} W(k, x)=1\right)$. However, it is not conserved [16],

$$
\begin{aligned}
\partial_{\mu}^{y} \tilde{T}^{\mu \nu}(y) & =\int \frac{\mathrm{d}^{4} k \mathrm{~d}^{4} x}{(2 \pi)^{4}} \mathrm{e}^{\mathrm{i} k(y-x) \star} \\
& \left(\partial_{\mu}^{x} W(k, x) \star T^{\mu \nu}(x)+W(k, x) \star \partial_{\mu}^{x} T^{\mu \nu}(x)\right) \\
& =\int \frac{\mathrm{d}^{4} k \mathrm{~d}^{4} x}{(2 \pi)^{4}} \mathrm{e}^{\mathrm{i} k(y-x) \star}
\end{aligned}
$$

\footnotetext{
${ }^{2}$ When considering $U_{\star}(N)$ gauge fields rather than $U_{\star}(1)$ fields as we do here, an additional trace appears in the product, i.e. $W \star T$ becomes $\operatorname{tr}(W \star T)$.
}

$$
\begin{aligned}
& \mathcal{P}_{\star}\left(\int_{0}^{1} \mathrm{~d} \sigma F_{\mu \alpha}(x+\sigma \theta k) \theta^{\alpha \beta}\left(\mathrm{i} k_{\beta}\right)\right. \\
& \left.\star W(k, x) \star T^{\mu \nu}(x)\right),
\end{aligned}
$$

where the star-commutator term arising from $\partial_{\mu} T^{\mu v}$ was canceled by part of the contribution coming from $\partial_{\mu} W$. The factor $i k_{\beta}$ can be pulled out of the integral by rewriting it as $\partial_{\beta}^{y}$, thus allowing for the definition of a gauge invariant, conserved (but no longer symmetric or traceless) EMT $\mathbf{T}^{\mu \nu}$ :

$$
\begin{aligned}
\mathbf{T}^{\mu \nu} & \equiv \tilde{T}^{\mu \nu}-\int \frac{\mathrm{d}^{4} k \mathrm{~d}^{4} x}{(2 \pi)^{4}} \mathrm{e}^{\mathrm{i} k(y-x)} \star \\
& \mathcal{P}_{\star}\left(\int_{0}^{1} \mathrm{~d} \sigma \theta^{\mu \alpha} F_{\alpha \beta}(x+\sigma \theta k) \star W(k, x) \star T^{\beta \nu}(x)\right) .
\end{aligned}
$$

The fact that this modified EMT is not traceless is actually not surprising since $\theta^{\mu \nu}$ is not dimensionless and thereby introduces a scale into the theory. However, sacrificing the symmetry of the EMT will only be worth the price, if the construction above also works when couplings to matter are considered. In the following sections, we show that this is not the case.

\section{Coupling to neutral matter fields}

One of the peculiarities of non-commutative space is that even neutral matter (such as neutrinos) can couple to $U_{\star}(1)$ gauge fields (photons) via star-commutators [26-28], i.e. the matter fields can transform with the adjoint representation [see Eq. (15) below] just like the gauge fields. In the following, we study the EMT of such neutral fields before discussing charged fields in Sect. 4.

\subsection{Complex scalar field}

Scalar field action: We consider an external $U_{\star}(1)$ gauge field $\left(A^{\mu}\right)$ and a complex scalar field $\phi$ in the adjoint representation, i.e. the infinitesimal gauge transformations read

$\delta_{\lambda} A_{\mu}=D_{\mu} \lambda, \quad \delta_{\lambda} \phi=-\mathrm{i} g\left[\phi^{\star}, \lambda\right], \quad \delta_{\lambda} \phi^{*}=-\mathrm{i} g\left[\phi^{*}, \lambda\right]$.

The minimal coupling of the field $\phi$ to the external gauge field $\left(A^{\mu}\right)$ is described by the action

$S[\phi ; A]=\frac{1}{2} \int \mathrm{d}^{4} x\left\{D_{\mu} \phi^{*}, D^{\mu} \phi\right\} \equiv \int \mathrm{d}^{4} x \mathcal{L}$,

where $D_{\mu} \cdot=\partial_{\mu} \cdot-\mathrm{i} g\left[A_{\mu} \stackrel{\star}{,} \cdot\right]$. (In this respect we note that $D_{\mu} \phi^{*} \equiv\left(D_{\mu} \phi\right)^{\dagger}=\partial_{\mu} \phi^{*}-\mathrm{i} g\left[A_{\mu} \stackrel{\star}{,} \phi^{*}\right]$.) As has been 
argued in Refs. [26,27], the fields in the adjoint representation carry zero $U_{\star}(1)$ charge. Concerning this point we note that the derivative $D_{\mu} \phi$ reduces in the commutative limit to $\partial_{\mu} \phi$, i.e. the coupling of neutral matter fields to gauge fields is only possible in a non-commutative setting.

Due to the invariance of the integral under a cyclic permutation of the factors in the star-product, the staranticommutator in the action (16) has no effect, but we choose to keep it in order to make manifest the symmetry under the exchange $\phi \leftrightarrow \phi^{*}$ in all expressions to be considered in the sequel. The equations of motion for the scalar field read

$$
\begin{gathered}
0=\frac{\delta S[\phi ; A]}{\delta \phi^{*}}=-D_{\mu} \star D^{\mu} \phi, \\
0=\frac{\delta S[\phi ; A]}{\delta \phi}=-D_{\mu} \star D^{\mu} \phi^{*},
\end{gathered}
$$

and we have

$$
J_{\mu} \equiv \frac{\delta S[\phi ; A]}{\delta A^{\mu}}=-\mathrm{i} g\left(\left[\phi^{\star}, D_{\mu} \phi^{*}\right]+\left(\phi \leftrightarrow \phi^{*}\right)\right) .
$$

This matter current (which vanishes in the commutative limit) is covariantly conserved, $D^{\mu} J_{\mu}=0$, as a consequence of the equations of motion (17) for $\phi$ and $\phi^{*}$.

The gauge transformation laws (15) imply that the covariant derivatives of $\phi$ and $\phi^{*}$ also transform covariantly, i.e. $\delta_{\lambda}\left(D_{\mu} \phi\right)=-\mathrm{i} g\left[D_{\mu} \phi \stackrel{\star}{,} \lambda\right]$ and analogously for $D_{\mu} \phi^{*}$. It follows that the Lagrangian density $\mathcal{L}$ in the action integral (16) transforms as $\delta_{\lambda} \mathcal{L}=-\mathrm{i} g[\mathcal{L}, \lambda]$ so that the action is gauge invariant. A short calculation using the Jacobi identity shows that the matter current (18) also transforms covariantly, $\delta_{\lambda} J_{\mu}=-\mathrm{i} g\left[J_{\mu} \stackrel{\star}{,} \lambda\right]$.

Next we turn to the EMT of the model described by the action (16). After coupling to an external gravitational field $\mathbf{g} \equiv\left(g_{\mu \nu}\right)$ we obtain the Einstein-Hilbert EMT in flat Moyal space:

$$
\begin{aligned}
T^{\mu \nu} & \left.\equiv\left(\frac{-2}{\sqrt{|g|}} \frac{\delta S[\phi ; A, \mathbf{g}]}{\delta g_{\mu \nu}}\right)\right|_{\mathbf{g}=\mathbb{1}} \\
& =\frac{1}{2}\left(\left\{D^{\mu} \phi^{*}, D^{v} \phi\right\}+\left(\phi \leftrightarrow \phi^{*}\right)-\delta^{\mu \nu}\left\{D_{\rho} \phi^{*}, D^{\rho} \phi\right\}\right) .
\end{aligned}
$$

From the equations of motion (17) it follows that

$$
D_{\mu} T^{\mu \nu}=-\frac{\mathrm{i} g}{2}\left(\left\{D_{\mu} \phi{ }^{\star}\left[F^{\mu \nu}, \phi^{*}\right]\right\}+\left(\phi \leftrightarrow \phi^{*}\right)\right) .
$$

For non-Abelian Yang-Mills theory in commutative space there would be a trace on the right hand side and the cyclic invariance of this trace would enable us to rewrite it in terms of the matter current $J_{\mu}$ as $\operatorname{Tr}\left(F_{\nu \mu} J^{\mu}\right)$ (i.e. we have a continuum version of the non-Abelian Lorentz-force equation). In the present case, however, all we can do is add and subtract the missing terms to arrive at

$$
\begin{aligned}
D_{\mu} T^{\mu \nu}= & \frac{1}{2}\left\{F^{\mu \nu}, J_{\mu}\right\} \\
& +\frac{\mathrm{i} g}{2}\left(\left[\phi^{\star},\left\{D_{\mu} \phi^{* \star}, F^{\mu \nu}\right\}\right]+\left(\phi \leftrightarrow \phi^{*}\right)\right) .
\end{aligned}
$$

In commutative space, the second term would vanish under the trace. In Moyal space, the cyclic invariance is only present under the integral $\int \mathrm{d}^{4} x$ (which in fact corresponds to a trace). However, integrating the above equation is not very helpful, since $\int \mathrm{d}^{4} x D_{\mu} T^{\mu \nu}=0$ for the left hand side, i.e. it is a surface term and we would not get any new information. In fact, as argued concerning Eq. (10), the second term on the right hand side of (21) also vanishes upon integration with $\int \mathrm{d}^{3} x$ in Minkowskian Moyal space with $\theta^{0 i}=0$ : we will come back to this point after adding the gauge field action.

Another observation is that, just like the Lagrangian density, the EMT is not gauge invariant for the same reason, i.e. due to lack of a trace (hence of the cyclic invariance). Instead, $T^{\mu \nu}$ transforms covariantly (as did its free gauge field counterpart discussed in the previous section):

$\delta_{\lambda} T^{\mu \nu}=-\mathrm{i} g\left[T^{\mu \nu}, \lambda\right]$.

Addition of the gauge field action: If we add to the action (16) a kinetic term for the gauge field so as to obtain the total action

$S_{\mathrm{tot}}[\phi, A]=\frac{1}{2} \int \mathrm{d}^{4} x\left\{D_{\mu} \phi^{* \star}, D^{\mu} \phi\right\}+\frac{1}{4} \int \mathrm{d}^{4} x F^{\mu \nu} \star F_{\mu \nu}$,

then the equation of motion of $A_{\mu}$ represents the non-commutative version of Maxwell's equations,

$D^{\mu} F_{\mu v}=J_{v}$

where the expression of $J_{v}$ in terms of $\phi$ and $\phi^{*}$ is given by Eq. (18). From (24) and the argumentation in Eq. (6) it follows that the current $J^{\mu}$ is covariantly conserved. Again, all fields and their covariant derivatives transform covariantly under gauge variations.

The associated Einstein-Hilbert EMT in flat Moyal space is a sum of expressions (7) and (19):

$$
\begin{aligned}
T_{\text {tot }}^{\mu \nu}= & \frac{1}{2}\left(\left\{D^{\mu} \phi \stackrel{\star}{,} D^{\nu} \phi^{*}\right\}+\left(\phi \leftrightarrow \phi^{*}\right)-\delta^{\mu \nu}\left\{D_{\rho} \phi^{* \star}, D^{\rho} \phi\right\}\right. \\
& \left.+\left\{F^{\mu \rho \star}, F_{\rho}^{v}\right\}-\frac{1}{2} \delta^{\mu \nu} F_{\rho \sigma} \star F^{\rho \sigma}\right) .
\end{aligned}
$$


Its covariant divergence can be obtained by using (24) which implies, for the gauge field EMT $T_{(A)}^{\mu \nu}$,

$D_{\mu} T_{(A)}^{\mu \nu}=-\frac{1}{2}\left\{F^{\mu \nu}, J_{\mu}\right\}$

and by adding (21) to this expression:

$D_{\mu} T_{\text {tot }}^{\mu \nu}=\frac{\mathrm{i} g}{2}\left(\left[\phi_{,}^{\star}\left\{D_{\mu} \phi^{*}, F^{\mu \nu}\right\}\right]+\left(\phi \leftrightarrow \phi^{*}\right)\right)$.

In commutative space, there would be a trace on the right hand side so that this expression would be zero and $T^{\mu v}$ would be conserved. (In fact, in that case we would also have a trace on the r.h.s. of (25), and $D_{\mu} T_{\text {tot }}^{\mu \nu}=\partial_{\mu} T_{\text {tot }}^{\mu \nu}$.) In the present case, however, we are once more lacking a trace to get rid of the r.h.s. In this respect we emphasize that according to the non-commutative generalization of Noether's theorem (see [29] and references therein), a continuous symmetry of the action does not generally imply a standard local conservation law for interacting theories: additional "source" terms (star-commutator terms which ultimately vanish under the space-time integral) generally appear. Actually, integration of (27) with $\int \mathrm{d}^{4} x$ yields trivially zero on both sides (since integration corresponds to a trace). In the Minkowskian version of Moyal space with non-commutativity parameters satisfying $\theta^{0 i}=0$, it suffices to integrate over $\int \mathrm{d}^{3} x$ to render the r.h.s. zero. In this case we have

$0=\int \mathrm{d}^{3} x D_{\mu} T_{\text {tot }}^{\mu \nu}=\int \mathrm{d}^{3} x \partial_{0} T_{\text {tot }}^{0 v}=\frac{\mathrm{d}}{\mathrm{d} t} \int \mathrm{d}^{3} x T_{\text {tot }}^{0 v}$,

which means that the four-momentum $P^{v} \equiv \int \mathrm{d}^{3} x T_{\text {tot }}^{0 v}$ is a conserved quantity [which is also gauge invariant by virtue of (8), (22)]. Of course the different contributions of $P^{v} \equiv$ $P_{\phi}^{v}+P_{A}^{v}$ are not conserved:

$\partial_{0} P_{\phi}^{v}=-\partial_{0} P_{A}^{v}=\int \mathrm{d}^{3} x F^{v \mu} J_{\mu}(\phi)$.

Restoring gauge invariance: In the present setting, we may follow the same strategy as in Sect. 2 and define an EMT $\tilde{T}^{\mu \nu}$ which is gauge invariant in analogy to expression (12):

$\tilde{T}_{\text {tot }}^{\mu v}(y) \equiv \int \frac{\mathrm{d}^{4} k \mathrm{~d}^{4} x}{(2 \pi)^{4}} \mathrm{e}^{\mathrm{i} k(y-x)} \star W(k, x) \star T_{\text {tot }}^{\mu \nu}(x)$.

For its divergence one obtains

$$
\begin{aligned}
& \partial_{\mu}^{y} \tilde{T}_{\text {tot }}^{\mu \nu}(y)=\int \frac{\mathrm{d}^{4} k d^{4} x}{(2 \pi)^{4}} \mathrm{e}^{\mathrm{i} k(y-x)} \star \\
& {\left[\mathcal{P}_{\star}\left(\int_{0}^{1} d \sigma\left(\mathrm{i} k_{\beta}\right) \theta^{\beta \alpha} F_{\alpha \mu}(x+\sigma \theta k) \star W(k, x) \star T^{\mu \nu}(x)\right)\right.}
\end{aligned}
$$

$$
\begin{aligned}
& +\frac{\mathrm{i} g}{2} W(k, x) \star\left(\left[\phi^{*},\left\{D_{\mu} \phi^{\star}, F^{\mu \nu}\right\}\right]\right. \\
& \left.\left.+\left[\phi^{\star}\left\{D_{\mu} \phi^{*}, F^{\mu \nu}\right\}\right]\right)\right] .
\end{aligned}
$$

The first term can be taken care of in the same way as in Eq. (14), but not so the second term. Thus, we are stuck with a small ( $\theta$-dependent) breaking of $\partial_{\mu} \tilde{T}^{\mu \nu}$, which of course vanishes in the commutative limit.

In Ref. [16] a redefinition of the EMT for a $\phi^{\star 4}$ theory which implies its conservation was discussed. However, in the present context the same strategy would destroy gauge covariance of $T_{\text {tot }}^{\mu \nu}$ making the construction of its gauge invariant counterpart via Wilson line impossible, as we will now show.

Since the additional terms on the r.h.s. of (27) are starcommutators it is generally possible to pull out one derivative by making use of the so-called $\star^{\prime}$-product (introduced in Refs. [30,31]),

$\left.\left(f \star^{\prime} g\right)(x) \equiv \frac{\sin \left(\frac{1}{2} \partial_{\mu}^{x} \theta^{\mu \nu} \partial_{\nu}^{y}\right)}{\frac{1}{2} \partial_{\rho}^{x} \theta^{\rho \sigma} \partial_{\sigma}^{y}} f(x) g(y)\right|_{x=y}$,

so that we may write

$[f \stackrel{\star}{,} g]=\mathrm{i} \theta^{\mu \nu} \partial_{\mu}\left(f \star^{\prime} \partial_{\nu} g\right)$.

Thus, a shift in the EMT ensuing its conservation can be made in principle, but at the cost of destroying its gauge covariance, i.e.

$$
\begin{aligned}
& \bar{T}_{\mathrm{tot}}^{\mu \nu} \equiv T_{\mathrm{tot}}^{\mu \nu}+a \frac{g}{2} \theta^{\mu \sigma}\left[\left(\phi^{*} \star^{\prime} \partial_{\sigma}\left\{D_{\rho} \phi, F^{\rho \nu}\right\}\right.\right. \\
& \left.+\phi \star^{\prime} \partial_{\sigma}\left\{D_{\rho} \phi^{*}, F^{\rho v}\right\}\right)-(1-a)\left(\partial_{\sigma} \phi^{*} \star^{\prime}\right. \\
& \left.\left.\left\{D_{\rho} \phi^{\star}, F^{\rho v}\right\}+\partial_{\sigma} \phi \star^{\prime}\left\{D_{\rho} \phi^{*}, F^{\rho v}\right\}\right)\right], \\
& D_{\mu} \bar{T}_{\text {tot }}^{\mu \nu}=0, \quad \bar{T}_{\text {tot }}^{\mu \nu} \neq \bar{T}_{\text {tot }}^{v \mu}, \quad \delta_{\lambda} \bar{T}_{\text {tot }}^{\mu \nu} \neq-\mathrm{i} g\left[\bar{T}_{\text {tot }}^{\mu \nu}{ }^{\star}, \lambda\right],
\end{aligned}
$$

where $a \in[0,1]$ is a free real parameter. Similarly, a redefinition achieving $\partial_{\mu} T_{\text {tot }}^{\mu \nu}=0$ could be made, but it would not gain us anything with respect to gauge invariance.

Thus, the best one can do is to use the gauge invariant expression (30) above with its modified conservation law (31). On the operator level, this means that only the trace over the divergence of the EMT (which is an operator in quantized space) is conserved, a fact which is obscured by the star-product prescription where the trace becomes an integral over space(-time). Therefore, it is not surprising that we have the local equation $\partial_{\mu} \tilde{T}^{\mu \nu} \neq 0$, as was already observed in the case of the $\phi^{\star 4}$-theory in Ref. [15]. We note that $\int \mathrm{d}^{4} y \partial_{\mu}^{y} \tilde{T}_{\text {tot }}^{\mu v}(y)=0=\int \mathrm{d}^{4} x \partial_{\mu}^{x} T_{\text {tot }}^{\mu \nu}(x)$ as can be checked explicitly by using the cyclic properties of the star-product 
under the integral, as well as $\int \mathrm{d}^{4} k \exp (\mathrm{i} k y)=(2 \pi)^{4} \delta^{4}(k)$ and $W(0, x)=1$.

In the next section, we show that one finds similar results when coupling to fermions instead of (or in addition to) scalars.

\subsection{Fermions}

We now consider an external $U_{\star}(1)$ gauge field and a neutral fermionic (Dirac) field (e.g. a non-commutative neutrino field [28]), i.e. fields in the adjoint representation with the gauge transformation properties

$$
\begin{array}{r}
\delta_{\lambda} \psi=-\mathrm{i} g\left[\psi^{\star}, \lambda\right], \quad \delta_{\lambda} \bar{\psi}=-\mathrm{i} g[\bar{\psi}, \lambda], \\
\delta_{\lambda} A_{\mu}=D_{\mu} \lambda \equiv \partial_{\mu} \lambda-\mathrm{i} g\left[A_{\mu}, \lambda\right] .
\end{array}
$$

The coupling of these fields is described by the action

$$
\begin{aligned}
S[\psi ; A] & =\int \mathrm{d}^{4} x \mathrm{i} \bar{\psi} \star \gamma^{\mu} D_{\mu} \psi, \quad \text { where } \\
D_{\mu} \psi & \equiv \partial_{\mu} \psi-\mathrm{i} g\left[A_{\mu} \stackrel{\star}{, \psi]},\right.
\end{aligned}
$$

and $D_{\mu} \bar{\psi} \equiv \partial_{\mu} \bar{\psi}-\mathrm{i} g\left[A_{\mu} \stackrel{\star}{,} \bar{\psi}\right]$, and where the square matrices $\gamma^{\mu}$ fulfill the Clifford algebra relation $\left\{\gamma^{\mu}, \gamma^{\nu}\right\}=2 \delta^{\mu \nu} \mathbb{1}$. The components $\psi_{\alpha}$ of the spinor field $\psi$ are considered to be anticommuting (i.e. Grassmann) variables. As for the scalar field case treated in the previous subsection, the coupling vanishes in the commutative limit where $D_{\mu} \psi$ reduces to $\partial_{\mu} \psi$.

The gauge transformation laws (35) imply

$\delta_{\lambda}\left(D_{\mu} \psi\right)=-\mathrm{i} g\left[D_{\mu} \psi \stackrel{\star}{,} \lambda\right], \quad \delta_{\lambda}\left(D_{\mu} \bar{\psi}\right)=-\mathrm{i} g\left[D_{\mu} \bar{\psi}, \lambda\right]$,

so that the Lagrangian density $\mathcal{L}$ of the model transforms as $\delta_{\lambda} \mathcal{L}=-\mathrm{i} g[\mathcal{L} \star \lambda]$, hence the action is gauge invariant.

The equations of motion for $\psi$ and $\bar{\psi}$ read

$\gamma^{\mu} D_{\mu} \psi=0=\left(D_{\mu} \bar{\psi}\right) \gamma^{\mu}$,

and the fermionic matter current is given by

$J^{\mu} \equiv \frac{\delta S}{\delta A_{\mu}}=-g \gamma_{\alpha \beta}^{\mu}\left\{\psi_{\beta} \stackrel{\star}{,} \bar{\psi}_{\alpha}\right\}$

where $\alpha, \beta$ denote the spinor indices. This current is covariantly conserved due to the equations of motion above (i.e. $D_{\mu} J^{\mu}=0$ ) and it transforms covariantly under gauge transformations: $\delta_{\lambda} J^{\mu}=-\mathrm{i} g\left[J^{\mu}, \lambda\right]$. Therefore, we also have $\delta_{\lambda}\left(D_{\mu} J^{\mu}\right)=-\mathrm{i} g\left[D_{\mu} J^{\mu} \stackrel{\star}{,}, \lambda\right]=0$. Furthermore, $J^{\mu}$ vanishes in the commutative limit.
The action (36) may also be written in the following more symmetric form by virtue of an integration by parts:

$$
\begin{aligned}
\int \mathrm{d}^{4} x \mathrm{i} \bar{\psi} \star \gamma^{\mu} \overleftrightarrow{D}_{\mu} \psi \equiv & \int \mathrm{d}^{4} x \frac{\mathrm{i}}{2}\left(\bar{\psi} \star \gamma^{\mu} D_{\mu} \psi\right. \\
& \left.-\left(D_{\mu} \bar{\psi}\right) \star \gamma^{\mu} \psi\right) .
\end{aligned}
$$

In analogy to the commutative case we obtain the EMT for the Dirac fields, ${ }^{3}$

$$
\begin{aligned}
T^{\mu \nu}= & \frac{\mathrm{i}}{4}\left[\left(\bar{\psi} \gamma^{\mu} \star D^{\nu} \psi-\left(D^{\mu} \bar{\psi}\right) \gamma^{\nu} \star \psi\right)+(\mu \leftrightarrow \nu)\right] \\
& -\mathrm{i} \delta^{\mu \nu} \bar{\psi} \star \gamma^{\rho} \overleftrightarrow{D}_{\rho} \psi \\
= & \frac{\mathrm{i}}{4}\left[\left(\bar{\psi} \gamma^{\mu} \star D^{\nu} \psi-\left(D^{\mu} \bar{\psi}\right) \star \gamma^{\nu} \psi\right)+(\mu \leftrightarrow \nu)\right],
\end{aligned}
$$

where the second line holds on-shell due to the equations of motion. Note that this tensor is hermitian as well as traceless on-shell. Furthermore, it represents a sum of products of bilinear quantities which transform covariantly, hence it also transforms covariantly under the gauge transformations (35),

$\delta_{\lambda} T^{\mu \nu}=-\mathrm{i} g\left[T^{\mu \nu} \stackrel{\star}{,}\right]$,

as can be checked straightforwardly.

To evaluate the covariant divergence of the EMT, we note that the equation of motion $\gamma^{v} D_{\nu} \psi=0$ implies $0=\left(\gamma^{\mu} D_{\mu}\right) \star\left(\gamma^{\nu} D_{\nu} \psi\right)=\gamma^{\mu} \gamma^{\nu} D_{\mu} D_{\nu} \psi$ : by decomposing $\gamma^{\mu} \gamma^{\nu}$ into its symmetric and antisymmetric parts and by using the commutation relation $\left[D_{\mu} \stackrel{\star}{,} D_{\nu}\right] \psi=-\mathrm{i} g\left[F_{\mu \nu}\right.$, $\psi]$ it now follows that

$\left.D^{\mu} D_{\mu} \psi=\frac{\mathrm{i}}{2} g \gamma^{\mu} \gamma^{\nu}\left[F_{\mu \nu}, *\right\}\right]$

The algebra of $\gamma$-matrices then yields the result

$$
\begin{aligned}
D_{\mu} T^{\mu \nu}= & g \bar{\psi} \star \gamma_{\mu} F^{\mu \nu} \star \psi-\frac{g}{2}\left\{F^{\mu \nu}, \bar{\psi} \star \gamma_{\mu} \psi\right\} \\
& +\frac{\mathrm{i}}{4}\left[\widetilde{F}^{\mu \nu}, J_{\mu}^{5}\right] \\
= & \frac{1}{2}\left\{F^{\mu \nu}, J_{\mu}\right\}+\frac{g}{2} \gamma_{\alpha \beta}^{\mu}\left(\left\{\bar{\psi}_{\alpha} \stackrel{\star}{,} F_{\mu}{ }^{\nu} \star \psi_{\beta}\right\}\right. \\
& \left.+\left\{\bar{\psi}_{\alpha} \star F_{\mu}{ }^{v} \star \psi_{\beta}\right\}\right)+\frac{\mathrm{i}}{4}\left[\widetilde{F}^{\mu \nu} \star J_{\mu}^{5}\right],
\end{aligned}
$$

where $\widetilde{F}^{\mu \nu} \equiv \frac{1}{2} \epsilon^{\mu \nu \rho \sigma} F_{\rho \sigma}$ and $J_{\mu}^{5} \equiv-g \bar{\psi} \star \gamma^{5} \gamma_{\mu} \psi$ denote the dual field strength tensor and what may be interpreted as a chiral current, respectively. The covariant divergence

\footnotetext{
${ }^{3}$ Its free counterpart (with coupling $g=0$ ) was previously constructed in Ref. [32] where the Sugawara form of the EMT for a free fermion in Moyal space was also established.
} 
of $T^{\mu \nu}$ again involves additional terms which would vanish under a trace in Yang-Mills theory, resp. an integral in Moyal space (the first term being compensated in the total EMT by the contribution (26) coming from the gauge field action). It is interesting to note that similar additional terms were found in the context of matrix models ${ }^{4}$ (cf. Appendix A.3 of Ref. [35]).

Once combined with the gauge field EMT as discussed in the previous subsection, one may again define the gauge invariant counterpart of the total EMT via Wilson line as in Eq. (30). Once more $\partial_{\mu} \tilde{T}_{\text {tot }}^{\mu \nu}$ involves breaking terms which depend on the non-commutativity parameters $\theta^{\mu v}$ and which cannot be absorbed into a redefined EMT in a gauge invariant way.

\section{Coupling to charged matter fields}

In this section, we discuss the coupling of gauge fields to charged scalars and fermions. In this case the EMT is found to be gauge invariant in general so that one does not have to resort to Wilson lines. Nonetheless we will not have the standard local conservation law for this EMT and the latter cannot be achieved while maintaining gauge invariance.

\subsection{Complex scalar field}

\subsubsection{Fundamental representation}

A complex scalar field in Moyal space can also be coupled to a gauge field via the covariant derivative $\bar{D}_{\mu} \phi \equiv \partial_{\mu} \phi-$ ig $A_{\mu} \star \phi$ and its hermitian conjugate expression, i.e.

$$
\begin{aligned}
& \bar{D}_{\mu} \phi \equiv \partial_{\mu} \phi-\mathrm{i} g A_{\mu} \star \phi, \\
& \bar{D}_{\mu}^{*} \phi^{*} \equiv\left(\bar{D}_{\mu} \phi\right)^{\dagger}=\partial_{\mu} \phi^{*}+\mathrm{i} g \phi^{*} \star A_{\mu}
\end{aligned}
$$

(where the latter derivative amounts to an action of $\partial_{\mu}+\mathrm{i} g A_{\mu}$ from the right). Thus, by contrast to the coupling discussed in the previous section, the basic covariant derivatives presently do not involve a commutator of fields: the field $\phi$ now transforms with the fundamental representation, i.e. we have the transformation rules

$$
\begin{gathered}
\delta_{\lambda} \phi=\mathrm{i} g \lambda \star \phi, \quad \delta_{\lambda} \phi^{*}=-\mathrm{i} g \phi^{*} \star \lambda, \\
\delta_{\lambda} A_{\mu}=D_{\mu} \lambda \equiv \partial_{\mu} \lambda-\mathrm{i} g\left[A_{\mu} \stackrel{\star}{,} \lambda\right]
\end{gathered}
$$

and thereby $\phi$ describes a charged field.

\footnotetext{
${ }^{4}$ Note that in the matrix model context, scalars appear as extra dimensions and do not exhibit such extra terms due to internal symmetries [33,34].
}

For a given external gauge field $A$, the action is now defined by

$S[\phi ; A]=\int \mathrm{d}^{4} x\left(\bar{D}_{\mu}^{*} \phi^{*}\right)\left(\bar{D}^{\mu} \phi\right)$,

and the associated equations of motion read

$$
\begin{gathered}
0=\frac{\delta S[\phi ; A]}{\delta \phi^{*}}=-\bar{D}_{\mu} \star \bar{D}^{\mu} \phi, \\
0=\frac{\delta S[\phi ; A]}{\delta \phi}=-\bar{D}_{\mu}^{*} \star \bar{D}^{* \mu} \phi^{*},
\end{gathered}
$$

while

$\left.J_{\mu} \equiv \frac{\delta S[\phi ; A]}{\delta A^{\mu}}=-\mathrm{i} g\left[\phi \star\left(\bar{D}_{\mu}^{*} \phi^{*}\right)-\left(\bar{D}_{\mu} \phi\right) \star \phi^{*}\right)\right]$.

The Lagrangian density $\mathcal{L} \equiv\left(\bar{D}_{\mu}^{*} \phi^{*}\right) \star\left(\bar{D}^{\mu} \phi\right)$ in the action integral (46) is invariant under the gauge transformations (45) which imply the transformation laws

$\delta_{\lambda}\left(\bar{D}_{\mu} \phi\right)=\mathrm{i} g \lambda \star \bar{D}_{\mu} \phi, \quad \delta_{\lambda}\left(\bar{D}_{\mu}^{*} \phi^{*}\right)=-\mathrm{i} g\left(\bar{D}_{\mu}^{*} \phi^{*}\right) \star \lambda$.

The matter current $J_{\mu}$ transforms covariantly, $\delta_{\lambda} J_{\mu}=$ $-\mathrm{i} g\left[J_{\mu} \stackrel{\star}{,} \lambda\right]$, and is covariantly conserved by virtue of the equations of motion of $\phi$ and $\phi^{*}$ :

$D^{\mu} J_{\mu} \equiv \partial^{\mu} J_{\mu}-\mathrm{i} g\left[A^{\mu}, J_{\mu}\right]=0$.

The transformation property of $J_{\mu}$ also leads to $\delta_{\lambda}\left(D^{\mu} J_{\mu}\right)=$ $-\mathrm{i} g\left[D^{\mu} J_{\mu} \stackrel{\star}{,} \lambda\right]=0$, which shows that the conservation law (50) is gauge invariant as well, and it thus provides a consistency check.

Before proceeding further, we note that the Lagrangian density is not uniquely defined. For instance, instead of $\mathcal{L} \equiv\left(\bar{D}_{\mu}^{*} \phi^{*}\right) \star\left(\bar{D}^{\mu} \phi\right)$ we can also consider the same expression with a different order of factors, $\mathcal{L}^{\prime} \equiv\left(\bar{D}^{\mu} \phi\right) \star\left(\bar{D}_{\mu}^{*} \phi^{*}\right)$ : both expressions differ by a total derivative, but $\mathcal{L}^{\prime}$ is not gauge invariant, $\delta_{\lambda} \mathcal{L}^{\prime}=\mathrm{i} g\left[\lambda \stackrel{{ }^{\star}}{\mathcal{L}^{\prime}}\right]$. A similar ambiguity occurs for the definition of the corresponding EMT which also represents a local, i.e. non-integrated quantity. As a matter of fact, we did not explicitly write any anticommutator in the action (46) in contrast to the scalar field model discussed in the previous section (where we had only one type of covariant derivative involving a commutator). This is a choice that we may make (because the anticommutator can be dropped under the integral in the action) and the motivation for the present choice is that the Lagrangian density and the corresponding EMT are now gauge invariant (in contrast to our previous scalar field model): the EMT of the model (46) reads 


$$
\begin{aligned}
T^{\mu \nu} \equiv & \left.\left(\frac{-2}{\sqrt{|g|}} \frac{\delta S[\phi ; A, \mathbf{g}]}{\delta g_{\mu \nu}}\right)\right|_{\mathbf{g}=\mathbb{1}}=\left(\bar{D}^{* \mu} \phi^{*}\right) \star\left(\bar{D}^{\nu} \phi\right) \\
& +(\mu \leftrightarrow v)-\delta^{\mu \nu}\left(\bar{D}_{\rho}^{*} \phi^{*}\right) \star\left(\bar{D}^{\rho} \phi\right),
\end{aligned}
$$

and we have

$$
\delta_{\lambda} T^{\mu \nu}=0,
$$

as follows readily from the transformation laws ${ }^{5}$ (49).

Finally, using

$$
\left[\bar{D}_{\mu} \stackrel{\star}{,} \bar{D}_{\nu}\right] \phi=-\mathrm{i} g F_{\mu \nu} \star \phi, \quad\left[\bar{D}_{\mu}^{*} \stackrel{\star}{,} \bar{D}_{\nu}^{*}\right] \phi^{*}=\mathrm{i} g \phi^{*} \star F_{\mu \nu},
$$

the divergence of the gauge invariant EMT (51) can be evaluated:

$$
\begin{aligned}
\partial_{\mu} T^{\mu v}= & \mathrm{i} g\left[\phi^{*} \star F^{\mu v} \star\left(\bar{D}_{\mu} \phi\right)-\left(\bar{D}_{\mu}^{*} \phi^{*}\right) \star F^{\mu v} \star \phi\right] \\
= & F^{\mu \nu} \star J_{\mu}+\mathrm{i} g\left[\phi^{*}, F^{\mu v} \star \bar{D}_{\mu} \phi\right] \\
& -\mathrm{i} g\left[\bar{D}_{\mu}^{*} \phi^{*}, F^{\mu v} \star \phi\right] .
\end{aligned}
$$

Once more, we have non-vanishing commutator terms ${ }^{6}$ due to a missing trace/integral (typical of non-commutative space) even in this simpler scalar field model. By adding the gauge field action and integrating with $\int \mathrm{d}^{3} x$ in Minkowskian Moyal space with $\theta^{0 i}=0$, we again find a conserved and gauge invariant four-momentum $\left(P^{v}\right)$ by virtue of Eqs. (28) and (52).

Notice that, in contrast to the case of neutral fields, no Wilson line construction is necessary to define a gauge invariant EMT, though in the present case the local conservation law of $T^{\mu \nu}$ is still broken by $\theta$-dependent terms. As mentioned in the previous section, in Ref. [16] a trick was used to eliminate similar (commutator) terms from $\partial_{\mu} T^{\mu v}$ in $\phi^{\star 4}$-theory in Moyal space (but without coupling to a gauge field). In that construction, one needs additionally the $\star^{\prime}$-product (32). We presently have

$$
\begin{aligned}
{\left[\phi^{*}{ }^{\star} F_{\mu \nu} \star \bar{D}^{\mu} \phi\right] } & =\mathrm{i} \theta^{\rho \sigma} \partial_{\rho}\left(\phi^{*} \star^{\prime} \partial_{\sigma}\left(F_{\mu \nu} \star \bar{D}^{\mu} \phi\right)\right) \\
& =\mathrm{i} \theta^{\rho \sigma} \partial_{\sigma}\left(\partial_{\rho} \phi^{*} \star^{\prime}\left(F_{\mu \nu} \star \bar{D}^{\mu} \phi\right)\right) ;
\end{aligned}
$$

and the expression

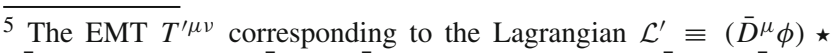
$\left(\bar{D}_{\mu}^{*} \phi^{*}\right)$, i.e. $T^{\prime \mu \nu}=\left(\bar{D}^{\mu} \phi\right) \star\left(\bar{D}^{* v} \phi^{*}\right)+(\mu \leftrightarrow v)-\delta^{\mu \nu}\left(\bar{D}^{\rho} \phi\right) \star\left(\bar{D}_{\rho}^{*} \phi^{*}\right)$ transforms covariantly under a gauge transformation (as does the one corresponding to the Lagrangian given by an anticommutator). Furthermore, its covariant derivative produces additional commutator terms similar to the ones present on the r.h.s. of Eq. (54) below.

${ }^{6}$ Note that $F^{\mu \nu} \star J_{\mu}=\frac{1}{2}\left\{F^{\mu \nu}, J_{\mu}\right\}+\frac{1}{2}\left[F^{\mu \nu} \star J_{\mu}\right]$ where the first term is the opposite of the covariant divergence of the gauge field EMT and where the second term is a star-commutator.
}

$$
\begin{aligned}
\bar{T}_{\mathrm{tot}}^{\mu v} \equiv & T_{\text {tot }}^{\mu \nu}+a g \theta^{\mu \sigma}\left(\phi^{*} \star^{\prime} \partial_{\sigma}\left(F^{\rho v} \star \bar{D}_{\rho} \phi\right)\right. \\
& \left.-\bar{D}_{\rho}^{*} \phi^{*} \star^{\prime} \partial_{\sigma}\left(F^{\rho v} \star \phi\right)\right) \\
& -(1-a) g \theta^{\mu \sigma}\left(\partial_{\sigma} \phi^{*} \star^{\prime}\left(F^{\rho v} \star \bar{D}_{\rho} \phi\right)\right. \\
& \left.-\partial_{\sigma}\left(\bar{D}_{\rho}^{*} \phi^{*}\right) \star^{\prime}\left(F^{\rho v} \star \phi\right)\right),
\end{aligned}
$$

with $a \in[0,1]$ a free parameter, is a locally conserved quantity. But the latter is no longer gauge invariant, nor is it symmetric.

\subsubsection{Antifundamental representation}

We note that there exists another possible choice for the transformation laws of fields and for the related covariant derivatives $[26,27]$. This choice is implemented by the replacement $\phi \leftrightarrow \phi^{*}$ (or the replacement $\bar{D}_{\mu} \leftrightarrow \bar{D}_{\mu}^{*}$ in integrands) and amounts to assuming that the scalar field $\phi$ is in the antifundamental representation rather than the fundamental representation (the latter corresponding to the transformation laws (45), the covariant derivatives (44) and the invariant action (46)); thus, we have the following expressions for the antifundamental representation:

$\delta_{\lambda} \phi=-\mathrm{i} g \phi \star \lambda, \quad \delta_{\lambda} \phi^{*}=\mathrm{i} g \lambda \star \phi^{*}$,

$\bar{D}_{\mu}^{*} \phi \equiv \partial_{\mu} \phi+\mathrm{i} g \phi \star A_{\mu}, \quad \bar{D}_{\mu} \phi^{*} \equiv \partial_{\mu} \phi^{*}-\mathrm{i} g A_{\mu} \star \phi^{*}$,

$S[\phi ; A]=\int \mathrm{d}^{4} x\left(\bar{D}_{\mu}^{*} \phi\right)\left(\bar{D}^{\mu} \phi^{*}\right)$.

Hence, we have a scalar field $\phi$ of opposite charge (but the additional change $g \rightarrow-g$ may be considered to switch signs and thereby have the same charge for $\phi$ as in the fundamental representation). This leads to the covariantly conserved current $\tilde{J}_{\mu}=\left.J_{\mu}\right|_{\phi \leftrightarrow \phi^{*}}$ and similarly to the EMT $\tilde{T}^{\mu \nu}$, which is related to the EMT $T^{\mu \nu}$ of the fundamental representation by the exchange $\phi \leftrightarrow \phi^{*}$.

\subsection{Fermions}

\subsubsection{Fundamental representation}

Finally, let us revisit the coupling of fermions to an external gauge field by considering the covariant derivative $\bar{D}_{\mu}$ :

$S[\psi ; A]=\int \mathrm{d}^{4} x \mathrm{i} \bar{\psi} \star \gamma^{\mu} \bar{D}_{\mu} \psi, \quad$ where

$\bar{D}_{\mu} \psi \equiv \partial_{\mu} \psi-\mathrm{i} g A_{\mu} \star \psi$

and $\bar{D}_{\mu}^{*} \bar{\psi} \equiv \partial_{\mu} \bar{\psi}+\mathrm{i} g \bar{\psi} \star A_{\mu}$. Thus, we now consider fermions in the fundamental representation $[36,37]$ (rather than the adjoint as in Sect. 3.2), the transformation laws being given by 


$$
\begin{gathered}
\delta_{\lambda} \psi=\mathrm{i} g \lambda \star \psi, \quad \delta_{\lambda} \bar{\psi}=-\mathrm{i} g \bar{\psi} \star \lambda, \\
\delta_{\lambda} A_{\mu}=\partial_{\mu} \lambda-\mathrm{i} g\left[A_{\mu}, \lambda\right] .
\end{gathered}
$$

These transformations leave the Lagrangian density $\mathcal{L} \equiv \mathrm{i} \bar{\psi} \star$ $\gamma^{\mu} \bar{D}_{\mu} \psi$ in the action functional (58) invariant and they imply

$\delta_{\lambda}\left(\bar{D}_{\mu} \psi\right)=\mathrm{i} g \lambda \star \bar{D}_{\mu} \psi, \quad \delta_{\lambda}\left(\bar{D}_{\mu}^{*} \bar{\psi}\right)=-\mathrm{i} g\left(\bar{D}_{\mu}^{*} \bar{\psi}\right) \star \lambda$.

The equations of motion of the present model read

$\gamma^{\mu} \bar{D}_{\mu} \psi=0, \quad\left(\bar{D}_{\mu}^{*} \bar{\psi}\right) \gamma^{\mu}=0$,

and the fermionic matter current is given by

$J^{\mu} \equiv \frac{\delta S}{\delta A_{\mu}}=-g \gamma_{\alpha \beta}^{\mu} \psi_{\beta} \star \bar{\psi}_{\alpha}$.

It is covariantly conserved by virtue of the equations of motion (61), i.e. $D_{\mu} J^{\mu} \equiv \partial_{\mu} J^{\mu}-\mathrm{i} g\left[A_{\mu}, J^{\mu}\right]=0$. Furthermore, it transforms covariantly under gauge transformations, $\delta_{\lambda} J^{\mu}=-\mathrm{i} g\left[J^{\mu}, \lambda\right]$. Thus, we also have $\delta_{\lambda}\left(D_{\mu} J^{\mu}\right)=0$.

The (on-shell) expression for the EMT of the fermion fields reads

$T^{\mu \nu}=\frac{\mathrm{i}}{4}\left[\left(\bar{\psi} \gamma^{\mu} \star \bar{D}^{\nu} \psi-\left(\bar{D}^{* \mu} \bar{\psi}\right) \star \gamma^{\nu} \psi\right)+(\mu \leftrightarrow \nu)\right]$.

It is traceless on-shell and (just like the Lagrangian density) it is invariant under the gauge transformations (59). Thus, no Wilson line construction is needed here either.

The divergence of this EMT can be determined by using the equations of motion:

$$
\begin{aligned}
\partial_{\mu} T^{\mu v}= & g \bar{\psi} \gamma_{\mu} \star F^{\mu v} \star \psi=F^{\mu v} \star J_{\mu} \\
& +g \gamma_{\alpha \beta}^{\mu}\left\{\bar{\psi}_{\alpha}, F_{\mu}{ }^{v} \star \psi_{\beta}\right\} .
\end{aligned}
$$

Once again, there is an additional term which would vanish under a trace, resp. an integral. Since the fermions are Grassmann variables, the additional term may be written in terms of a $\star^{\prime}$-product as

$\left\{\bar{\psi}_{\alpha} \stackrel{\star}{,} F_{\mu}{ }^{\nu} \star \psi_{\beta}\right\}=i \theta^{\rho \sigma} \partial_{\rho} \bar{\psi}_{\alpha} \star^{\prime} \partial_{\sigma}\left(F_{\mu}{ }^{\nu} \star \psi_{\beta}\right)$.

This allows for a redefinition of $T_{\text {tot }}^{\mu \nu}$ which is conserved but not gauge invariant.

Before closing this section, let us see what happens if we consider the EMT corresponding to the classically equivalent Lagrangian $\mathcal{L}^{\prime} \equiv-\mathrm{i} \gamma_{\alpha \beta}^{\mu}\left(\bar{D}_{\mu} \psi_{\beta}\right) \star \bar{\psi}_{\alpha}$ :

$$
\begin{aligned}
T^{\prime \mu \nu}= & \frac{-\mathrm{i}}{4}\left[\left(\gamma_{\alpha \beta}^{\mu}\left(\bar{D}^{\nu} \psi_{\beta}\right) \star \bar{\psi}_{\alpha}-\gamma_{\alpha \beta}^{\mu} \psi_{\beta} \star\left(\bar{D}^{* \nu} \bar{\psi}_{\alpha}\right)\right)\right. \\
& +(\mu \leftrightarrow v)] .
\end{aligned}
$$

This expression is not gauge invariant but transforms covariantly: $\delta_{\lambda} T^{\prime \mu \nu}=-\mathrm{i} g\left[T^{\prime \mu \nu}, \lambda\right]$. For the covariant divergence of the EMT (66) we get a result which bears some resemblance with the expression (43) obtained for fermion fields in the adjoint representation,

$D_{\mu} T^{\prime \mu \nu}=\frac{1}{2}\left\{F^{\mu \nu}, J_{\mu}\right\}-\frac{\mathrm{i}}{4}\left[\widetilde{F}^{\mu \nu}, J_{\mu}^{5}\right]$,

where $\widetilde{F}^{\mu \nu} \equiv \frac{1}{2} \epsilon^{\mu \nu \rho \sigma} F_{\rho \sigma}$ and $J_{\mu}^{5} \equiv-g\left(\gamma^{5} \gamma_{\mu}\right)_{\alpha \beta} \psi_{\beta} \star \bar{\psi}_{\alpha}$. Thus, we again have an additional commutator term in the covariant divergence of the EMT.

\subsubsection{Antifundamental representation}

Roughly speaking, the interchange of $\psi_{\alpha}$ and $\bar{\psi}_{\beta}$ (or of $\bar{D}$ and $\bar{D}^{*}$ in integrands) in the previous expressions allows us to obtain the fermion field (of opposite charge) in the antifundamental representation:

$\delta_{\lambda} \psi=-\mathrm{i} g \psi \star \lambda, \quad \delta_{\lambda} \bar{\psi}=\mathrm{i} g \lambda \star \bar{\psi}$,

$\bar{D}_{\mu}^{*} \psi \equiv \partial_{\mu} \psi+\mathrm{i} g \psi \star A_{\mu}, \quad \bar{D}_{\mu} \bar{\psi} \equiv \partial_{\mu} \bar{\psi}-\mathrm{i} g A_{\mu} \star \bar{\psi}$,

$S[\psi ; A]=\int \mathrm{d}^{4} x \mathrm{i} \bar{\psi} \star \gamma^{\mu} \bar{D}_{\mu}^{*} \psi$.

In this case, the covariantly conserved current takes the familiar form

$\tilde{J}^{\mu}=-g \bar{\psi} \star \gamma^{\mu} \psi$,

i.e. Eq. (62) with $\bar{\psi}_{\alpha}$ and $\psi_{\beta}$ exchanged. The resulting expression for the EMT in the antifundamental representation is obtained by exchanging $\bar{D}_{\mu}$ and $\bar{D}_{\mu}^{*}$ in expression (63): $\tilde{T}^{\mu \nu}=\frac{\mathrm{i}}{4}\left[\left(\bar{\psi} \gamma^{\mu} \star \bar{D}^{* \nu} \psi-\left(\bar{D}^{\mu} \bar{\psi}\right) \star \gamma^{\nu} \psi\right)+(\mu \leftrightarrow \nu)\right]$. Just like the Lagrangian density $\tilde{\mathcal{L}} \equiv \mathrm{i} \bar{\psi} \star \gamma^{\mu} \bar{D}_{\mu}^{*} \psi$, this tensor transforms covariantly with the adjoint representation under gauge transformations. For the covariant divergence of the EMT we have results which are similar to those holding in the fundamental representation.

\section{Conclusion}

According to the non-commutative generalization of Noether's theorem [29], some extra $\theta$-dependent terms ("source"/ star-commutator terms) generally appear in the local conservation law for the EMT for interacting theories. The lack of gauge invariance and local conservation of the EMT is not surprising since the EMT represents, very much like the Lagrangian density, a non-integrated expression and it is only the integral over Moyal space which ensures the cyclic invariance of factors in star-products, and thereby the vanishing of star-commutator terms. 
In the present paper, we have explicitly shown (for complex scalars as well as for fermions coupled to gauge fields) that the standard local conservation law of the EMT $T^{\mu \nu}$ is always modified due to non-commutative effects and that $T^{\mu \nu}$ can always be redefined so as to be conserved, but that the so defined EMT is not gauge invariant. (Yet, for dynamical matter and gauge fields we always have a conserved and gauge invariant four-momentum with components $P^{v}=\int \mathrm{d}^{3} x T^{0 v}$.)

More specifically, we discussed two possible couplings of scalars and fermions to gauge fields corresponding to neutral and charged matter, respectively: In the first case, the basic EMT transforms covariantly and its gauge invariant counterpart could be constructed by using the non-commutative generalization of a Wilson line. In the second case (for which there exist two variants, namely the fundamental and the antifundamental representations), the freedom in the definition of the EMT allows for the choice of a gauge covariant or a gauge invariant tensor. For all cases we found that the consideration of the $\star^{\prime}$-product allows one to achieve the standard local conservation law for the EMT, but at the expense of losing gauge invariance (and symmetry). We note that the tools employed here are also those which are generally considered for the quantization; e.g. see Refs. [23,30,38].

Our systematic study is tantamount to a proof that it is not possible to construct a conserved and gauge invariant (and symmetric) EMT for spin 0 and spin $1 / 2$ matter fields coupled to a $U_{\star}(1)$ gauge field in Moyal space. ${ }^{7}$ Yet, in all cases the total energy-momentum $P^{v} \equiv \int \mathrm{d}^{3} x T_{\text {tot }}^{0 v}$ of the system represents a conserved and gauge invariant quantity. In practice, the formulation of classical as well as quantum field theories in flat space primarily relies on the conserved charges $P^{v}$, so that the problematic properties of the EMT that we discussed can somehow be circumvented. However, the situation is quite different in curved space, where one has to couple the EMT to a metric field while taking into account the related non-commutativities.

Acknowledgments D. B. wishes to thank H. Steinacker for pointing out to us Ref. [35]. Furthermore, we wish to thank the anonymous referees for their valuable comments which helped us to clarify several points.

Open Access This article is distributed under the terms of the Creative Commons Attribution 4.0 International License (http://creativecomm ons.org/licenses/by/4.0/), which permits unrestricted use, distribution, and reproduction in any medium, provided you give appropriate credit to the original author(s) and the source, provide a link to the Creative Commons license, and indicate if changes were made. Funded by SCOAP ${ }^{3}$.

\footnotetext{
7 An indirect cure of the problems for the case of neutral scalars appears to be the passage to the matrix model framework since these scalars appear naturally as extra dimensions in this framework and the extra terms we found in the conservation law of the EMT are not present there due to internal symmetries [33,34].
}

\section{References}

1. H.J. Groenewold, On the principles of elementary quantum mechanics. Physica 12, 405-460 (1946)

2. J.E. Moyal, Quantum mechanics as a statistical theory. Proc. Camb. Philos. Soc. 45, 99-124 (1949)

3. R.J. Szabo, Quantum field theory on noncommutative spaces. Phys. Rep. 378, 207-299 (2003). arXiv:hep-th/0109162

4. V. Rivasseau, Non-commutative renormalization, in Séminaire Poincaré X (2007)—Espaces Quantiques, ed. by B. Duplantier, V. Rivasseau (Birkhäuser, Boston, 2007). arXiv:0705.0705 [hep-th]

5. D.N. Blaschke, E. Kronberger, R.I.P. Sedmik, M. Wohlgenannt, Gauge theories on deformed spaces. SIGMA 6, 062 (2010). arXiv: 1004.2127 [hep-th]

6. H. Grosse, G. Lechner, T. Ludwig, R. Verch, Wick rotation for quantum field theories on degenerate Moyal space(-time). J. Math. Phys. 54, 022307 (2013). arXiv:1111.6856 [hep-th]

7. S. Minwalla, M. Van Raamsdonk, N. Seiberg, Noncommutative perturbative dynamics. JHEP 02, 020 (2000). arXiv:hep-th/9912072

8. A. Matusis, L. Susskind, N. Toumbas, The IR/UV connection in the non-commutative gauge theories. JHEP 12, 002 (2000). arXiv:hep-th/0002075

9. H. Grosse, R. Wulkenhaar, Renormalisation of $\phi^{4}$ theory on noncommutative $\mathbb{R}^{2}$ in the matrix base. JHEP 12, 019 (2003). arXiv:hep-th/0307017

10. H. Grosse, R. Wulkenhaar, Renormalisation of $\phi^{4}$ theory on noncommutative $\mathbb{R}^{4}$ in the matrix base. Commun. Math. Phys. 256, 305-374 (2005). arXiv:hep-th/0401128

11. R. Gurau, J. Magnen, V. Rivasseau, A. Tanasa, A translationinvariant renormalizable non-commutative scalar model. Commun. Math. Phys. 287, 275-290 (2009). arXiv:0802.0791 [math-ph]

12. H. Grosse, R. Wulkenhaar, Self-dual noncommutative $\phi^{4}$-theory in four dimensions is a non-perturbatively solvable and non-trivial quantum field theory. Commun. Math. Phys. 329, 1069-1130 (2014). arXiv:1205.0465 [math-ph]

13. A. Micu, M.M. Sheikh Jabbari, Noncommutative $\Phi^{4}$ theory at two loops. JHEP 01, 025 (2001). arXiv:hep-th/0008057

14. T. Pengpan, X. Xiong, A note on the noncommutative Wess-Zumino model. Phys. Rev. D 63, 085012 (2001). arXiv:hep-th/0009070

15. A. Gerhold, J. Grimstrup, H. Grosse, L. Popp, M. Schweda, R. Wulkenhaar, The energy-momentum tensor on noncommutative spaces: some pedagogical comments. arXiv:hep-th/0012112

16. M. Abou-Zeid, H. Dorn, Comments on the energy momentum tensor in noncommutative field theories. Phys. Lett. B 514, 183-188 (2001). arXiv:hep-th/0104244

17. J.M. Grimstrup, B. Kloibock, L. Popp, V. Putz, M. Schweda, M. Wickenhauser, The energy momentum tensor in noncommutative gauge field models. Int. J. Mod. Phys. A 19, 5615-5624 (2004). arXiv:hep-th/0210288

18. A.K. Das, J. Frenkel, On the energy momentum tensor in noncommutative gauge theories. Phys. Rev. D 67, 067701 (2003). arXiv:hep-th/0212122

19. D. Bahns, S. Doplicher, K. Fredenhagen, G. Piacitelli, On the unitarity problem in space/time noncommutative theories. Phys. Lett. B 533, 178-181 (2002). arXiv:hep-th/0201222

20. T.C. Adorno, D.M. Gitman, A.E. Shabad, D.V. Vassilevich, Classical noncommutative electrodynamics with external source. Phys. Rev. D 84, 065003 (2011). arXiv:1106.0639 [hep-th]

21. P. Sikivie, N. Weiss, Classical Yang-Mills theory in the presence of external sources. Phys. Rev. D 18, 3809 (1978)

22. H. Balasin, D.N. Blaschke, F. Gieres, M. Schweda, Wong's equations and charged relativistic particles in non-commutative space. SIGMA 10, 099 (2014). arXiv:1403.0255 [hep-th] 
23. D.J. Gross, A. Hashimoto, N. Itzhaki, Observables of noncommutative gauge theories. Adv. Theor. Math. Phys. 4, 893-928 (2000). arXiv:hep-th/0008075

24. D. Berenstein, R.G. Leigh, Observations on noncommutative field theories in coordinate space. arXiv:hep-th/0102158

25. N. Ishibashi, S. Iso, H. Kawai, Y. Kitazawa, Wilson loops in noncommutative Yang-Mills. Nucl. Phys. B 573, 573-593 (2000). arXiv:hep-th/9910004

26. M. Hayakawa, Perturbative analysis on infrared aspects of noncommutative QED on $\mathbb{R}^{4}$. Phys. Lett. B 478, 394-400 (2000). arXiv:hep-th/9912094

27. M. Chaichian, P. Prešnajder, M.M. Sheikh-Jabbari, A. Tureanu, Noncommutative gauge field theories: a no-go theorem. Phys. Lett. B 526, 132-136 (2002). arXiv:hep-th/0107037

28. P. Schupp, J. Trampetic, J. Wess, G. Raffelt, The photon neutrino interaction in non-commutative gauge field theory and astrophysical bounds. Eur. Phys. J. C 36, 405-410 (2004). arXiv:hep-ph/0212292

29. J. Zahn, Wirkungs- und Lokalitätsprinzip für nichtkommutative skalare Feldtheorien. Master's thesis, Universität Hamburg (2003)

30. H. Liu, J. Michelson, *-Trek: the one loop $N=4$ noncommutative SYM action. Nucl. Phys. B 614, 279-304 (2001). arXiv:hep-th/0008205
31. T. Mehen, M.B. Wise, Generalized $\star$-products, Wilson lines and the solution of the Seiberg-Witten equations. JHEP 12, 008 (2000). arXiv:hep-th/0010204

32. M. Ghasemkhani, Noncommutative Sugawara construction. arXiv:1407.3831 [hep-th]

33. Y. Okawa, H. Ooguri, Energy momentum tensors in matrix theory and in noncommutative gauge theories. arXiv:hep-th/0103124

34. H. Steinacker, Emergent gravity and noncommutative branes from Yang-Mills matrix models. Nucl. Phys. B 810, 1-39 (2009). arXiv:0806.2032 [hep-th]

35. A. Polychronakos, H. Steinacker, J. Zahn, Brane compactifications and 4-dimensional geometry in the IKKT model. Nucl. Phys. B 875, 566-598 (2013). arXiv:1302.3707 [hep-th]

36. J.M. Gracia-Bondia, C.P. Martín, Chiral gauge anomalies on noncommutative $\mathbb{R}^{4}$. Phys. Lett. B 479, 321-328 (2000). arXiv:hep-th/0002171

37. E.F. Moreno, F.A. Schaposnik, Wess-Zumino-Witten and fermion models in noncommutative space. Nucl. Phys. B 596, 439-458 (2001). arXiv:hep-th/0008118

38. S. Bellucci, I. Buchbinder, V. Krykhtin, Renormalization of the energy momentum tensor in noncommutative scalar field theory. Nucl. Phys. B 665, 402-424 (2003). arXiv:hep-th/0303186 